\section{RSP}

http://www.rsp.fsp.usp.br/
Revista de Saúde Pública

\title{
Retos sobre la carga epidemiológica y económica para diabetes e hipertensión en México
}

Armando Arredondo', Emanuel Orozco', Jaqueline Alcalde-Rabanal', Juan Navarro", Alejandra Azar'

Instituto Nacional de Salud Pública. Cuernavaca, Morelos, Mexico

" Universidade Federal da Bahia. Instituto de Saúde Coletiva. Salvador, BA, Brasil

\section{RESUMEN}

OBJETIVO: Analizar la carga epidemiológica y económica de la demanda de servicios de salud por diabetes e hipertensión en México.

MÉTODOS: Investigación evaluativa basada en un estudio de series de tiempo que tomó como universo de estudio la población asegurada y no asegurada que demanda servicios de salud a las tres principales instituciones del Sistema de Salud en México: Secretaría de Salud, Instituto Mexicano del Seguro Social, e Instituto de Servicios y Seguridad Social para los Trabajadores del Estado. El método de costeo tomó como base las técnicas de instrumentación y de consenso por manejo de caso promedio. Para estimar los cambios epidemiológicos y requerimientos financieros, se integró una serie de tiempos de casos observados para diabetes e hipertensión 1994-2013. Se desarrollaron modelos probabilísticos basados en la técnica de Box-Jenkins para el periodo 2013-2018 con intervalos del 95\% de confianza y $\mathrm{p}<0.05$.

RESULTADOS: Comparando resultados de 2013 versus 2018, en las cinco regiones, se observaron diferentes tendencias incrementales de $14 \%-17 \%$ en cambios epidemiológicos y de $58 \%-66 \%$ en la carga económica para ambas enfermedades.

Correspondencia:

Armando Arredondo

Instituto Nacional de Salud Pública Av. Universidad, 655 Col. Santa María Ahuacatitlán

CP 62100 Cuernavaca, Morelos, México

E-mail: armando.arredondo@insp.mx

Recibido: 29 sept 2016

Aprobado: 19 jun 2017

Cómo citar: Arredondo A, Orozco E, Alcalde-Rabanal J, Navarro J, Azar A. Retos sobre la carga epidemiológica y económica para diabetes e hipertensión en México. Rev Saude Publica. 2018;52:23.

Copyright: Este es un artículo de el acceso abierto distribuido bajo la términos de la licencia Atribución Creative Commons, lo que permite el uso ilimitado, distribución y reproducción en cualquier medio, siempre que el autor y la fuente los originales se acreditan.
CONCLUSIONES: Si los factores de riesgo y los diferentes modelos de atención permanecieran como están actualmente en las tres instituciones de análisis, las consecuencias financieras serían de mayor impacto para el Instituto Mexicano del Seguro Social, siguiendo en orden de importancia el Instituto de Servicios y Seguridad Social para los Trabajadores del Estado y finalmente para la Secretaría de Salud. Los requerimientos financieros para ambas enfermedades representarán aproximadamente del 13\%-15\% del presupuesto total asignado para población no asegurada y el 15\%-17\% para población asegurada dependiendo de la región.

DESCRIPTORES: Diabetes Mellitus, epidemiología. Hipertensión, epidemiología. Necesidades y Demandas de Servicios de Salud, economía. Financiación de la Atención de la Salud. 


\section{INTRODUCCIÓN}

En el marco de una cobertura universal efectiva, el incremento de los costos de servicios de salud para la atención de enfermedades no transmisibles, la necesidad de incrementar la inversión, la desprotección financiera del usuario de servicios de salud y la urgencia de cambios en los métodos de asignación de recursos en el sector salud, han suscitado múltiples inquietudes en los tomadores de decisiones ${ }^{1,2}$. La demanda de atención a la salud no disminuye y el elevado costo de las distintas alternativas de acción en este período de transiciones en todos los ámbitos impone a los presupuestos nacionales y estatales una pesada carga que los gobiernos están tratando de reducir³.

Las enfermedades cardiovasculares en las personas con diabetes mellitus inician más tempranamente, y suelen presentarse con síntomas y signos atípicos ${ }^{4}$. Las complicaciones de las enfermedades crónicas como la diabetes y la hipertensión se incrementan de manera importante. La inversión de los sistemas de salud para tratar la diabetes debe contemplar no solo el manejo de la enfermedad sino además sus complicaciones. La diabetes es una emergencia mundial debido a los excesivos gastos que atentan sobre la sostenibilidad de los sistemas de salud. Las complicaciones de las enfermedades no transmisibles causan costos considerables: se ha demostrado que la nefropatía diabética es la complicación más costosa de la diabetes en la región de las Américas.

En términos económicos, el significado de los cambios en el perfil epidemiológico y demográfico es un incremento en la demanda de atención de padecimientos costosos (tratamiento de enfermedades crónico degenerativas y accidentes). Eso competirá con el presupuesto asignado para el tratamiento de enfermedades infecto-contagiosas aún sin resolver ${ }^{5,6}$. Así, habrá una necesidad de desarrollar y aplicar instrumentos de análisis que permitan revalorar las prioridades de salud y establecer acciones estratégicas para la asignación, utilización y organización óptima de los recursos financieros del sector salud ${ }^{7-9}$.

El presente artículo tuvo por objetivo analizar la carga epidemiológica y económica de la demanda de servicios de salud por diabetes e hipertensión en México. Se analizaron los cambios epidemiológicos esperados para el período 2013-2018, así como los requerimientos financieros para las dos demandas de estudio en el Instituto Mexicano del Seguro Social (IMSS), la Secretaría de Salud (SSA) y el Instituto de Servicios y Seguridad Social para los Trabajadores del Estado (ISSSTE) a nivel de cada sistema estatal de salud.

\section{MÉTODOS}

Investigación evaluativa basada en un estudio de series de tiempo en México para el período 2013-2018. Los estados seleccionados fueron Jalisco, Hidalgo, Morelos, Sinaloa y Yucatán. Los estados estudiados mostraron relativa heterogeneidad para los indicadores de marginación, aseguramiento público, rezago epidemiológico e índice de población originaria, en tanto indicadores macro de desarrollo socioeconómico ${ }^{10,11}$ (Tabla 1). Los índices de rezago epidemiológico y población originaria representaron mayores retos en todos los casos por coincidir el mayor índice de marginación con el mayor rezago epidemiológico, lo cual suele ser más acuciante en población originaria.

Tabla 1. Características sociodemográficas de los estados seleccionados. Cuernavaca, 2016.

\begin{tabular}{lccccc}
\hline Estado & Población total & Índice de Marginación & Índice de Aseguramiento Público & Índice de Rezago Epidemiológico & Índice de Población Indígena \\
\hline Sinaloa & $2,966,321$ & Bajo & Alto & Bajo & Bajo \\
Morelos & $1,903,811$ & Medio & Medio & Medio & Majo \\
Hidalgo & $2,858,359$ & Alto & Medio & Bajo & Medio \\
Jalisco & $7,844,830$ & Bajo & Alto & Alto & Bajo \\
Yucatán & $2,395,272$ & Alto & Medio & Alto \\
\hline
\end{tabular}

Fuentes: Consejo Nacional de Población - Instituto Nacional de Estadística y Geografía, 2000-2015. Secretaría de Salud, Anuarios Estadísticos sobre Daños a la Salud, 1998-2015. Información para la Rendición de Cuentas, México, 2006-2015. Censo de Población $2010-2015$. 
Las instituciones de estudio incluyeron el IMSS, la SSA y el ISSSTE. La demanda anual de servicios de salud para hipertensión y diabetes se obtuvo a partir del número de casos en control para diabetes e hipertensión reportados por tipo de institución. Dicha información se obtuvo del boletín de estadísticas sobre daños a la salud del Sistema Nacional de Salud, 1994-2013 ${ }^{12}$.

Los costos directos de la atención se obtuvieron a partir del manejo de caso promedio estandarizado y ajustado por tipo de institución. El método de costeo se basó en la metodología de grupos de diagnóstico relacionado (variante de GRD [Group of Related Diagnostic] ajustada para México $)^{13}$ y apoyados en la técnica de instrumentación. Una vez integrados los equipos de trabajo y con la selección de las enfermedades a costear (diabetes e hipertensión), se procedió a trabajar con la técnica de consenso para determinar los manejos de caso promedio. Para ello, se discutió a manera de prueba piloto los instrumentos de recolección que de acuerdo a la historia natural de la enfermedad y a la experiencia laboral del equipo multidisciplinario fueron rediseñados para captar los distintos procesos, procedimientos y recursos ( funciones de producción e insumos), así como todas las actividades ex-ante y ex-post de cada función de producción para otorgar el servicio que requiere cada patología. La participación de los médicos fue fundamental para la determinación de los distintos procesos de atención médica y por el hecho de que se discutió la calidad de la atención que se brinda en el hospital y en los diferentes niveles de atención desde la perspectiva del proveedor.

Los instrumentos de recolección de datos para cada función de producción contemplaron distintas categorías de análisis de insumos y costos: infraestructura, recursos humanos en horas médico, horas enfermera y horas personal administrativo, estudios de laboratorio, estudios complementarios, promedio de estancia-día-cama, componentes de terapia medicamentosa-curativa, componentes de terapia intensiva, servicios contratados, servicios generales, otros recursos, procesos y procedimientos y observaciones. Los insumos y costos se concentraron en matrices de costeo por tipo de enfermedad y tipo de institución.

Se trabajó con esta técnica para establecer consenso y estandarizar la metodología de obtención de funciones de producción e insumos. Los profesionales de las distintas áreas (clínica, epidemiológica y administrativa) tuvieron una participación activa y determinante en el manejo de caso promedio por enfermedad. Todo integrante del equipo de trabajo tuvo como experiencia mínima cinco años en la práctica clínico-administrativa.

Para determinar los requerimientos financieros de casos esperados, se realizó una serie de tiempos 1994-2013. Se diseñaron modelos autorregresivos utilizando el software Stat Graphics bajo la técnica de Box-Jenkins ${ }^{14}$ y con intervalos del 95\% de confianza y $\mathrm{p}<0.05$. El método propuesto se justificó porque las observaciones de casos mostraron ser altamente correlacionadas. Eso, porque se dispuso de datos registrados para diabetes e hipertensión con la calidad y cantidad requeridas para aplicar dicha técnica. Además, es uno de los métodos más recomendados para proyecciones a mediano y largo plazo en el análisis de problemas crónicos de salud pública. El desarrollo de los modelos consideró cada una de las siguientes etapas y pasos metodológicos:

\section{Etapa 1: Identificación}

- Identificación del modelo tentativo de la serie de tiempo para usar en la predicción.

- Revisión de la calidad de la información y número de observaciones para diabetes e hipertensión.

- Análisis de autocorrelación de las observaciones históricas.

\section{Etapa 2: Estimación}

- Determinación de estimadores de los parámetros, usando el criterio de mínimos cuadrados.

- Aplicación de un procedimiento iterativo de búsqueda de una función de suma de cuadrados, especificando previamente los estimadores preliminares de los parámetros desconocidos. 


\section{Etapa 3: Chequeo del Diagnóstico}

- Prueba de adecuación, después de que los modelos fueron ajustados a los datos.

- Análisis de la diferencia entre lo observado y lo esperado en casos de diabetes e hipertensión.

- Aplicación de la Chi cuadrada de Box Pierce.

\section{Etapa 4: Predicción}

- Selección y diseño del modelo definitivo.

- Procesamiento de datos.

- Predicción de valores esperados definitivos para la serie de tiempo, tanto en diabetes como en hipertensión

La selección de modelos y ecuaciones de predicción se diseñaron con casos observados hasta el 2012. Una vez que se elaboró un informe de los resultados, se ratificó actualizar las proyecciones con los casos observados hasta diciembre de 2012, proyectando para el período 2013-2018. Se utilizaron estos resultados para proceder al análisis de implicaciones financieras de cambios epidemiológicos para hipertensión y diabetes en cada institución y sistema estatal de salud bajo estudio.

Para estimar las consecuencias financieras de los cambios en las demandas por tipo de institución y enfermedad, se aplicó un índice inflacionario proyectado al 2013-2018, tomando como base el índice de precios al consumidor del Banco de México ${ }^{15}$. Los resultados fueron presentados en Moneda Nacional y dólares de EUA, al tipo de cambio en junio de 2015 y con una tasa de cambio de 1 \$EUA $=14.35$ \$Mex.

\section{RESULTADOS}

Los resultados para el caso de diabetes y de hipertensión fueron similares en el desarrollo del modelo excepto en el número de casos por tipo de enfermedad, como se aprecia más adelante. Los datos históricos fueron el número de casos mensuales de diabetes en el período de 1996 a 2012. Los números de casos mensuales fueron denotados por Y1, Y2,...,Y84. El número de casos siguió un patrón con una tendencia fuerte a incrementarse hasta el año de 2000, disminuyendo bruscamente en 2001 e incrementándose de nuevo en 2002, con picos irregulares hasta el 2012. El coeficiente de la pendiente en el análisis de la tendencia fue positivo y significativamente diferente de cero $(t=6.76)$. La serie de tiempo fue descrita por el factor tendencia y el número de casos de diabetes tendió a incrementarse.

Sobre la función de autocorrelación de la serie original, la serie no fue estacionaria, debido a que la función de autocorrelación disminuyó lentamente. Después de aplicar la transformación de una diferencia no estacional, la serie de tiempo parece ser estacionaria y puede explicarse por la transformación Zt = Yt - Yt-1 es decir, una diferencia no estacional.

Parece ser que la función de autocorrelación corta a la línea que representa a la frontera de la significancia estadística de diferencia de cero después del lag 1 , y que la función de autocorrelación parcial muere rápidamente. Eso indica la necesidad de incluir un operador de movimiento promedio de orden 1. Otra interpretación de ambas funciones es que la función de autocorrelación muere rápidamente y que la función de autocorrelación parcial corta en el lag 1. Así, la serie de tiempo podría ser explicada por un operador autorregresivo de orden 1.

Como resultado del análisis de las funciones de autocorrelación y autocorrelación parcial, se propusieron los siguientes modelos:

Modelo 1 - Operador de movimiento promedio de orden 1:

$$
\mathrm{Z}_{\tau}=\delta=\varepsilon_{\tau}-\theta_{1} \varepsilon_{\tau}-1
$$


Modelo 2 - Operador autorregresivo de orden 1:

$$
\mathrm{Z}_{\tau}=\delta+\phi_{1} \mathrm{Z}_{\tau}-1
$$

Los resultados obtenidos de los dos modelos fueron presentados en la Tabla 1. Se incluyeron los estimadores de los parámetros en cada modelo, la estadística t, la estadística chi-cuadrada de Box-Pierce en los primeros 20 residuales, y se indicó los lags en los que los residuales tenían autocorrelaciones significativamente diferentes de cero. Se presentó el valor de la desviación estándar (s) para cada modelo. Los dos modelos ajustaron bien a los datos, y no tuvieron autocorrelaciones de los residuales significativamente diferentes de cero. Se seleccionó el modelo 2 como el modelo definitivo porque fue el de menor error estándar (Tabla 2).

Con los resultados de este ajuste, se demostró que el operador autorregresivo de orden 1 describió adecuadamente los casos de diabetes mellitus, ya que la diferencia entre lo observado y estimado fue pequeña. Como el valor de la pendiente de la serie resultó significativamente diferente de cero $(t=6.76)$, un término constante fue usado al ajustar el modelo. Eso coincide con los juicios de los planeadores en salud, quienes consideran que las enfermedades crónicas tienden a incrementarse, es decir, que la tasa de tendencia incremental continuará en el futuro a corto, mediano y largo plazo. Por esta razón, ambos modelos incluyeron ese término. El modelo adecuado fue el Modelo 2, con la ecuación de predicción.

$$
\gamma+{ }_{\tau}=\delta+\gamma_{\tau-13}+\beta_{1,12} \varepsilon_{\tau-13}-\theta_{1} \varepsilon_{\tau-1}+\theta_{1} \theta_{1,12} \varepsilon_{\tau-13}+\varepsilon_{\tau}
$$

Donde:

$\gamma+{ }_{\tau}=$ Promedio anual de casos esperados

$\delta=$ Factor residual de autorcorrelacion parcial

$\gamma_{\tau-13}=$ Valor histórico de la serie de años previos a la predicción

$\beta_{1,12} \varepsilon_{\tau-13}=$ Coeficiente de autocorrelación parcial de la serie de tiempo

$\theta_{1} \varepsilon_{\tau-1}=$ Número de casos por tipo de estimador (A, B or C)

$\theta_{1} \theta_{1,12} \varepsilon_{\tau-13}=$ Valor promedio de la serie por autocorrelación total

$\varepsilon_{\tau}=$ Valor crítico de la desviación estándar

En todos los estados se observaron tendencias epidemiológicas de casos observados y esperados para diabetes e hipertensión crecientes constantes, con picos irregulares antes del 2012, sobre todo en Jalisco, donde se observó la mayor carga epidemiológica en términos absolutos (Tabla 3). A diferencia de la diabetes, en el caso de la hipertensión, el primer lugar en términos del número de casos, lo compartirán Jalisco y Sinaloa. Morelos, Yucatán e Hidalgo presentaron tendencias constantes, ligeramente crecientes y sin picos irregulares.

Tabla 2. Resultados de pruebas estadísticas de modelos de estimación de casos de diabetes e hipertensión. Cuernavaca, 2016.

\begin{tabular}{lcc}
\hline Variable & Modelo 1 & Modelo 2 \\
\hline Número de diferencias no estacionarias & 1 & 1 \\
Número de diferencias estacionarias & 0 & 0 \\
Número de Parámetros & 3 & 2 \\
Estimador A & $0.7689(4.01)$ & $0.2579(2.43)$ \\
Estimador B & $0.5536(2.23)$ & $-0.2723(-2.54)$ \\
Estimador C & 0.9123 & 0.8599 \\
Chi-cuadrada de Box-Pierce & 14.67 & 22.25 \\
Desviación Estándar & 26.9 & 21.22 \\
Correlaciones diferentes de cero & 0 & 0 \\
\hline
\end{tabular}


Tabla 3. Número de casos esperados por tipo de enfermedad a nivel estatal (casos en valor absoluto). Cuernavaca, $2013-2018$.

\begin{tabular}{|c|c|c|c|c|c|c|c|}
\hline \multirow{2}{*}{ Enfermedad/Estado } & \multicolumn{6}{|c|}{ Número de casos por año } & \multirow{2}{*}{$\mathbf{p}$} \\
\hline & 2013 & 2014 & 2015 & 2016 & 2017 & 2018 & \\
\hline \multicolumn{8}{|c|}{ Diabetes } \\
\hline Sinaloa & 16,126 & 15,524 & 17,457 & 16,677 & 18,270 & 18,496 & \multirow{4}{*}{$<0.01$} \\
\hline IC95\%* & $15,926-16,326$ & $15,114-15,934$ & $16,937-17,977$ & $15,997-17,357$ & $17,530-19,010$ & $17,646-19,346$ & \\
\hline Morelos & 10,618 & 10,803 & 11,660 & 11,982 & 12,681 & 12,975 & \\
\hline IC95\%* & $10,418-10,818$ & $10,393-11,213$ & $11,140-12,180$ & $11,302-12,662$ & $11,941-13,421$ & $12,125-13,825$ & \\
\hline Hidalgo & 9,232 & 9,605 & 9,864 & 10,355 & 10,686 & 11,124 & \multirow[t]{2}{*}{$<0.01$} \\
\hline IC95\%* & $9,032-9,432$ & $9,195-10,015$ & $9,344-10,384$ & $9,675-11,035$ & $9,946-11,426$ & $10,274-11,974$ & \\
\hline Jalisco & 19,154 & 20,794 & 21,454 & 21,996 & 22,352 & 22,666 & \multirow[t]{2}{*}{$<0.01$} \\
\hline IC95\%* & $18,954-19,354$ & $20,384-21,204$ & $20,934-21,974$ & $21,316-22,676$ & $21,612-23,092$ & $21,816-23,516$ & \\
\hline Yucatán & 6,174 & 6,409 & 6,382 & 6,700 & 6,980 & 7,172 & \multirow[t]{2}{*}{$<0.01$} \\
\hline IC95\%* & $5,974-6,374$ & $5,999-6,819$ & $5,862-6,902$ & $6,020-7,380$ & $6,240-7,720$ & $6,322-8,022$ & \\
\hline \multicolumn{8}{|c|}{ Hipertensión } \\
\hline Sinaloa & 28,017 & 29,673 & 31,406 & 33,018 & 34,303 & 35,424 & $<0.01$ \\
\hline IC95\%* & $27,817-28,217$ & $29,263-30,083$ & $30,886-31,926$ & $32,338-33,698$ & $33,563-35,043$ & $34,574-33,274$ & \\
\hline Morelos & 15,353 & 15,645 & 16,010 & 16,196 & 17,392 & 17,619 & $<0.01$ \\
\hline IC95\%* & $15,153-15,553$ & $15,235-16,055$ & $15,490-16,530$ & $15,516-16,876$ & $16,652-18,132$ & $16,769-18,469$ & \\
\hline Hidalgo & 9,992 & 10,246 & 10,422 & 11,107 & 11,441 & 11,748 & $<0.01$ \\
\hline IC95\%* & $9,792-10,192$ & $9,836-10,656$ & $9,902-10,942$ & $10,427-11,787$ & $10,701-12,181$ & $10,898-12,598$ & \\
\hline Jalisco & 26,982 & 30,851 & 31,169 & 31,658 & 32,116 & 32,477 & $<0.01$ \\
\hline IC95\%* & $26,782-27,182$ & $30,441-31,261$ & $30,649-31,689$ & $30,978-32,338$ & $31,376-32,856$ & $31,627-33,327$ & \\
\hline Yucatán & 8,953 & 9,222 & 9,465 & 9,620 & 9,785 & 10,022 & $<0.01$ \\
\hline IC95\%* & $8,753-9,153$ & $8,812-9,632$ & $8,945-9,985$ & $8,940-10,300$ & $9,045-10,525$ & $9,172-10,872$ & \\
\hline
\end{tabular}

* Intervalos al 95\% de confianza con prueba estadística de Box-Pierce.

Las tendencias irregulares se observaron más en el caso de Jalisco y Sinaloa para la primera parte del periodo.

Se observaron tendencias crecientes constantes en los requerimientos financieros en todos los estados. Los incrementos fueron en un rango de $8 \%$ a 40\% dependiendo del año de análisis y del estado. El estado que presentó los mayores requerimientos financieros fue Jalisco, seguido de Sinaloa, Morelos, Hidalgo y finalmente Yucatán con una carga económica casi a la mitad del penúltimo estado (Hidalgo). Comparando el estado de mayor carga económica (Jalisco) con el estado de menor carga económica (Yucatán), la diferencia fue más o menos de cinco a uno.

La mayor carga económica la presentó el IMSS, seguido de la SSA y finalmente el ISSSTE. Los resultados detallando los costos y consecuencias financieras atribuibles a la hipertensión aparecen en las tablas de anexos. Importante resaltar que en las tres instituciones se presentaron tendencias crecientes constantes para cada uno de los años del periodo de estimación.

A diferencia de la carga económica de la diabetes, la hipertensión presentó la mayor carga económica para el caso de Sinaloa seguidos de Jalisco, Morelos, Hidalgo y Yucatán en orden decreciente. Comparando los rangos de impacto económico por estado, Sinaloa como de alto impacto y Yucatán como de bajo impacto, la diferencia fue más o menos de tres a uno. Las tendencias incrementales sí fueron similares a las de Diabetes en un rango del 8.0\% al 40.0\% dependiendo del periodo de análisis.

Las tendencias económicas y epidemiológicas se pueden observar gráficamente para el periodo 2012-2018 (Figuras 1-4). Para el caso de diabetes, quedó evidente la diferencia entre los cinco estados, y la ausencia de periodos irregulares sin traslapes entre los estados, quedando en orden de importancia Jalisco, Sinaloa, Morelos, Hidalgo y Yucatán. 


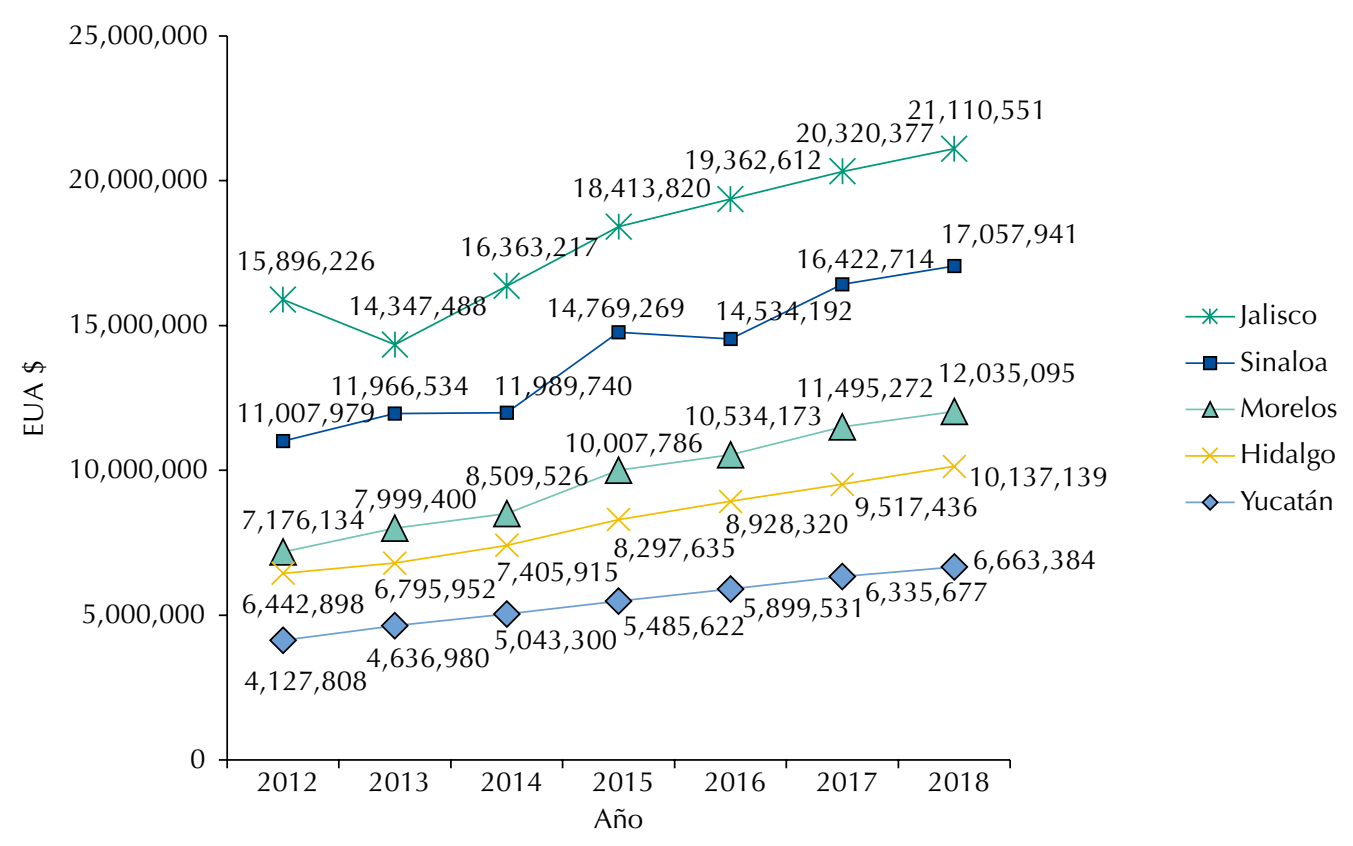

Tasa de cambio 14.35 \$MEX por 1 \$EUA (Periodo de referencia junio de 2015).

Figura 1. Costos totales de diabetes en diferentes estados (en \$ EUA). Cuernavaca, 2012-2018.

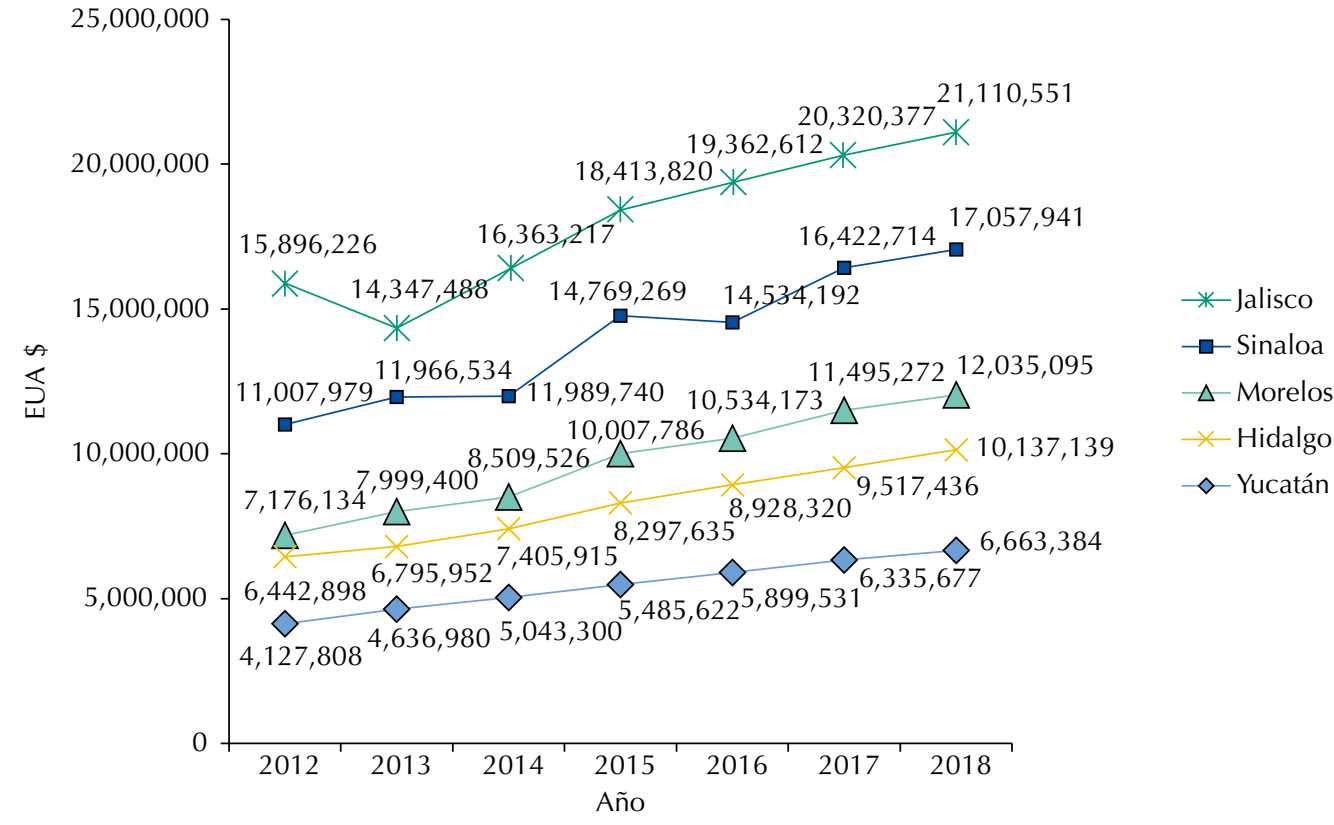

Tasa de cambio 14.35 \$MEX por 1 \$EUA (Periodo de referencia junio de 2015).

Figura 2. Costos totales de hipertensión en diferentes estados (en \$ EUA). Cuernavaca, 2012-2018.

Se notó la diferencia de la tendencia de combinación de carga epidemiológica y económica para la diabetes mencionada al principio contra la tendencia en tasas de prevalencia. En efecto, mientras que, en la combinación de tendencias epidemiológicas y carga económica el primer lugar lo ocupó Jalisco, seguido de Sinaloa, Morelos, Hidalgo y Yucatán en orden decreciente, en el caso de la prevalencia, el primer lugar lo ocupa Yucatán, seguido por Hidalgo, Sinaloa, Morelos y Jalisco. Se invierten totalmente la tendencia en los estados, de manera que Yucatán quedó en primer lugar y Jalisco en último lugar por orden de importancia en el impacto.

Para el caso de hipertensión, mientras que, en la combinación de carga epidemiológica y económica el mayor impacto fue para Sinaloa y Jalisco, seguido de Yucatán, Hidalgo y 


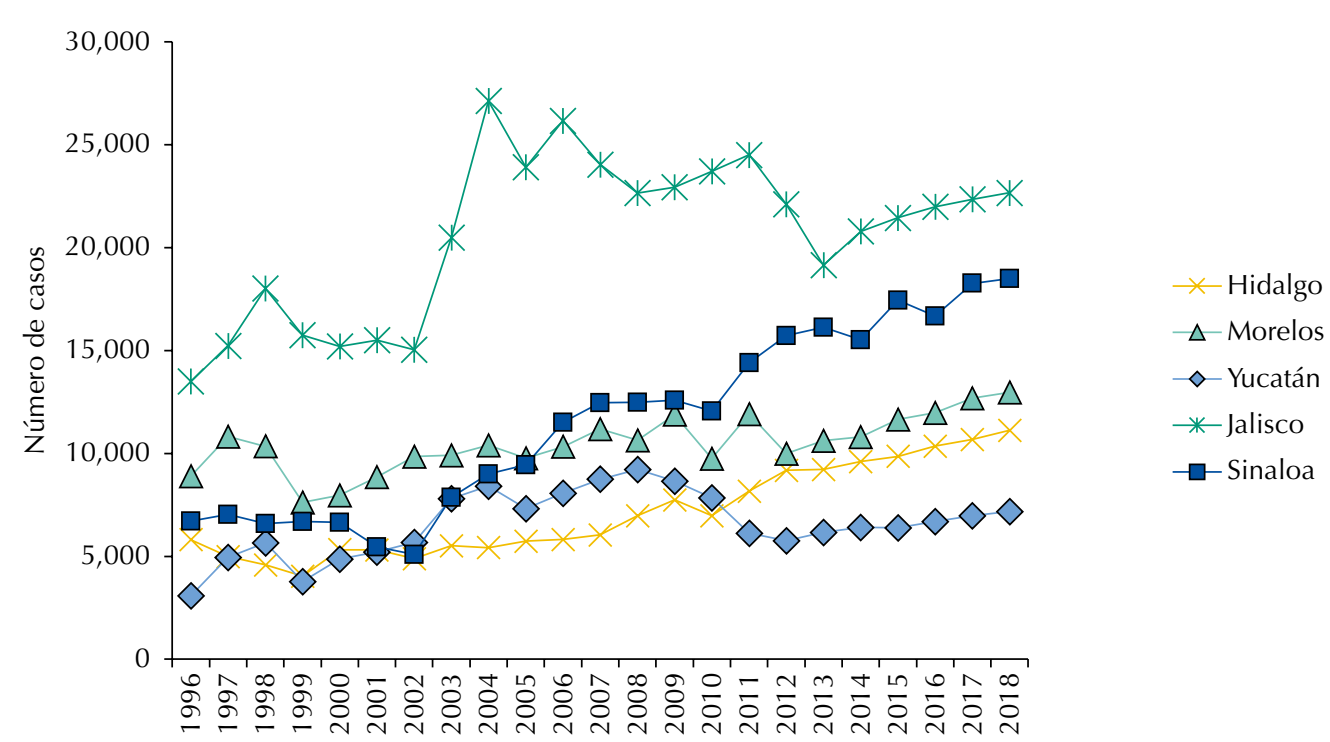

Año

Figura 3. Tendencia de casos observados 1996-2012 y casos esperados 2013-2018 para diabetes en los estados bajo estudio. Cuernavaca, 2016.

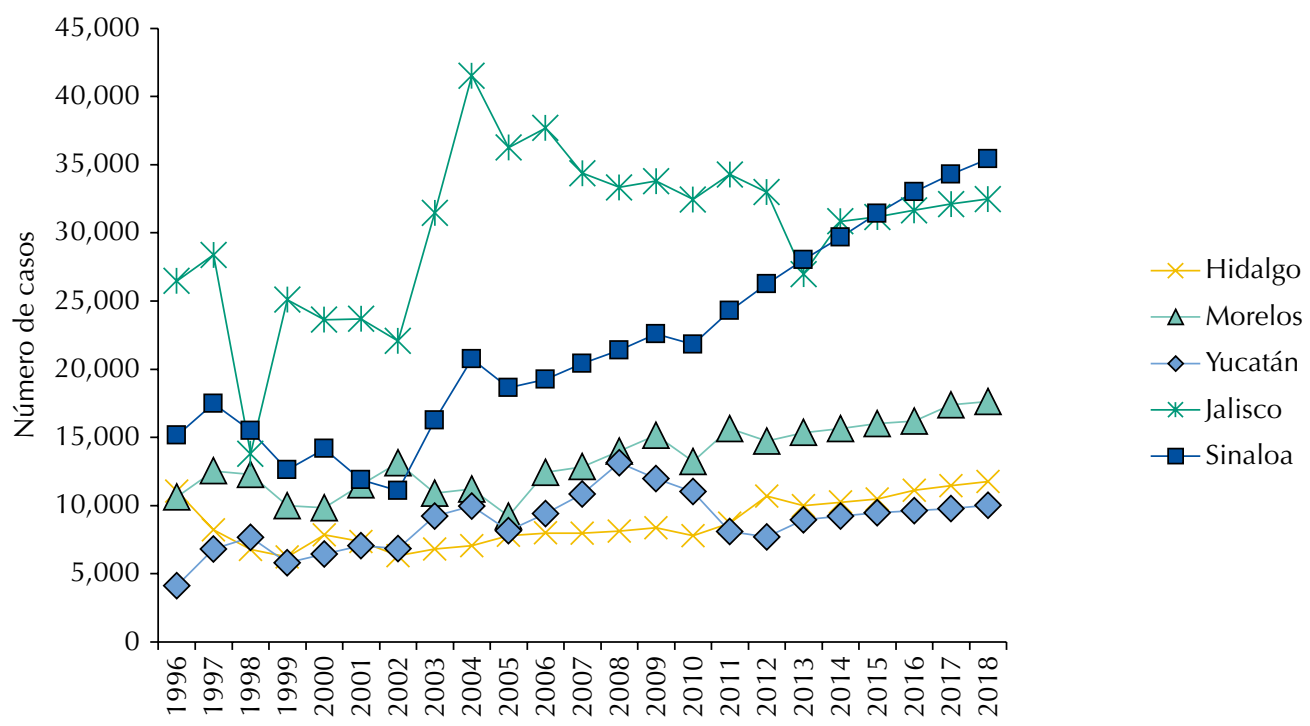

Año

Figura 4. Tendencia de casos observados 1996-2012 y casos esperados 2013-2018 para hipertensión en los estados bajo estudio. Cuernavaca, 2016.

Morelos en orden decreciente, en el caso del impacto por prevalencia existen similitudes en las tendencias. En efecto, la mayor prevalencia fue para Sinaloa seguida de Hidalgo, Jalisco, Yucatán y Morelos en orden decreciente. En ambos análisis, las tendencias de mayor y menor impacto fueron para Sinaloa y Morelos, respectivamente.

\section{DISCUSIÓN}

Las tendencias de nuestros hallazgos en términos de tendencias epidemiológicas y de impacto económico, coinciden de manera importante con otros estudios o análisis similares ${ }^{16-20}$. Sus análisis coinciden en la necesidad de incorporar la evaluación económica al interior del sector salud y de organizar con mayor eficiencia la producción de servicios priorizando de acuerdo a las necesidades reales de la población que los va a consumir. Las tendencias incrementales constantes se justifican por varias razones: la diversificación de la demanda 
por presiones en cambios epidemiológicos esperados, la escasez de recursos financieros y las variadas e infinitas necesidades de salud que se generarán con los cambios importantes del perfil epidemiológico para los años futuros en la mayoría de los países en desarrollo, principalmente de América Latina ${ }^{21,22}$.

El cambio en los perfiles epidemiológicos y el consecuente incremento en la demanda de recursos destinados a la atención de estas enfermedades crónicas sugieren fuertemente la necesidad de ajustar el modelo de atención de modo que los casos sean, cada vez más, atendidos de forma ambulatoria. El objetivo es disminuir la aparición de complicaciones que requieren manejo hospitalario. Una característica esencial del modelo ajustado sería incrementar el valor que la atención ambulatoria tiene para el paciente. Ese objetivo se encuentra en relación clara con la racionalidad económica de la de eficiencia, al igual que resultó en estudios similares de costos en salud para enfermedades crónicas ${ }^{17,23}$.

Los sistemas de aseguramiento se financian con recursos públicos procedentes de los impuestos. Dada la crisis económica mundial y sus efectos en la salud ${ }^{24}$, los presupuestos de salud, particularmente para México y la mayoría de los países de América Latina, han sufrido un decremento importante en los últimos años ${ }^{25}$. Más que nunca, se requiere de instituciones eficientes en la asignación y manejo de los recursos. Igualmente, se requiere que el uso eficiente de recursos garantice la factibilidad financiera de estrategias que minimicen los costos de atención vinculados a padecimientos crónicos, es decir orientar los recursos hacia acciones básicamente preventivas y de promoción de la salud. En tal sentido, la diabetes y la hipertensión requieren de implementación de modelos de atención orientados al empoderamiento y participación activa de los usuarios para que el autocuidado favorezca la identificación de complicaciones de forma temprana. Estos modelos que involucran al paciente y su familia en el cuidado de su salud son altamente efectivos porque pueden reportar de manera temprana la aparición de una complicación. En consecuencia, se puede disminuir considerablemente las complicaciones. Ello tendrá consecuencias favorables en la disminución o mayor control de los costos de atención vinculados a estos padecimientos y sus complicaciones. Se requiere de fortalecimiento del primer nivel de atención para el impulso y fortalecimiento de un modelo centrado en la prevención y promoción de la salud.

Los resultados de nuestro estudio muestran la relevancia de incorporar aspectos epidemiológicos y económicos a la perspectiva clínica en tanto propuesta integral para el análisis y evaluación del desempeño del sistema de salud en el contexto de las reformas del sector. El desarrollo de estudios de investigación evaluativa que integren una valoración económica con valoraciones clínica y epidemiológica, se torna relevante a partir de expectativas empíricas. A medida que avancen los proyectos de reforma de la salud, el costo de prestar servicios únicamente a la demanda de casos hospitalarios de enfermedades crónico-degenerativas será más alto con relación al costo de proporcionar servicio a la demanda de casos ambulatorios y hospitalarios de enfermedades infecciosas. Ello implica que a mayor avance de la transición epidemiológica, mayores consecuencias financieras en la producción de servicios de atención médica para demandas futuras sobre enfermedades como diabetes e hipertensión.

Los cambios observados y esperados en el perfil epidemiológico de las enfermedades crónicas respecto a las infecciosas conducirán a una competencia financiera en el uso de los recursos. La asignación de recursos financieros para producir servicios dirigidos a enfermedades crónicas estará afectada por la producción de servicios para enfermedades infecciosas. La competencia interna en el uso y asignación de los recursos económicos requiere del conocimiento aproximado de los requerimientos financieros para producir los servicios que se demandarán en el corto y mediano plazos. En este sentido, la producción y financiamiento de servicios de salud, requerirá incorporar indicadores clínicos, epidemiológicos y económicos integrando dichos indicadores bajo el criterio de eficiencia dependiendo del contexto de cada sistema estatal de salud.

Los resultados permiten hacer cambios importantes en la asignación de recursos y en ajustes al modelo de atención desde los sistemas estatales de salud en función de las variables de rezago epidemiológico, peso relativo de las comunidades indígenas, incorporación de 
aspectos culturales en propuestas de modelos de prevención y promoción tomando en cuenta hábitos y costumbres a nivel de cada región. Este análisis diferencial tendrá efectos importantes en el fenómeno de competencia interna en el uso y asignación de recursos en diferentes escenarios. Por ejemplo, en Jalisco y Sinaloa, en los que se observan tendencias crecientes de diabetes e hipertensión más elevadas que el resto de los estados incluidos en el estudio. Eso nos hace inferir tendencias de crecimiento desiguales en diabetes e hipertensión en los estados y que supone tres escenarios: estados como alto crecimiento (Jalisco y Sinaloa), estados con mediano crecimiento (Yucatán) y estados con bajo crecimiento (Hidalgo y Morelos). Estos escenarios requerirán de investigación para explicar los factores que influyen en el menor o mayor crecimiento de las enfermedades crónicas y de soporte para implementar estrategias integradas en un modelo de atención que permita frenar el crecimiento de este tipo de padecimientos.

La principal limitación en estudios de serie de tiempo se refiere a la disponibilidad de datos de calidad y cantidad en el número de observaciones. Sin embargo, se contó con un número adecuado de observaciones en este estudio para poder hacer las estimaciones con un alto grado de confiabilidad. A medida que nos vayamos moviendo al último año del periodo, tanto la carga epidemiológica como la económica puede tener menor precisión y certidumbre; por eso se recomienda hacer un monitoreo para actualizar la serie de tiempos y sus respectivas carga epidemiológica y económica al menos cada dos años. Otra limitación es que la carga económica estimada podrá variar en la medida que haya cambios en la tecnología médica y medicamentos para el manejo de ambas enfermedades, por posibles cambios en el protocolo o bien por posibles cambios en los programas de salud, o bien por cambios inflacionarios en el índice de precios de insumos. Se espera que estos cambios no sucedan en periodos de cinco años por lo que el monitoreo bianual podría controlar estas limitaciones.

Se puede replicar el análisis de niveles y tendencias epidemiológicas y económicas esperadas, en términos de frecuencias relativas, a nivel nacional, estatal/provincial o institucional (instituciones para asegurados e instituciones para la asistencia pública). La misma tendencia de frecuencias relativas se puede aplicar para el análisis de otras enfermedades crónicas de cualquier país con cambios epidemiológicos similares a México, ya que las enfermedades de estudio fueron seleccionadas bajo el criterio de trazadores de la transición epidemiológica. También se puede aplicar hacia otros niveles de intervención, enfermedades u otras instituciones del sector salud es el referente a las frecuencias relativas que corresponden a cada función de producción sobre el costo de manejo de intervenciones.

Los cambios en el perfil epidemiológico de países de América Latina plantean que uno de los mayores desafíos es el económico. Tanto la competencia interna como los patrones de asignación de recursos se verán afectados directamente por los cambios epidemiológicos esperados. Los costos de proveer servicios dirigidos a nuevas y más demandas de enfermedades crónicas, además de competir con las demandas clásicas de enfermedades infecciosas, obligan a cambios importantes en los patrones de asignación de recursos. Los costos y las consecuencias financieras del cambio en el perfil epidemiológico son elementos analíticos que deberán tomarse en cuenta en la planeación de actuales y futuros planes o programas de salud a nivel regional, nacional y estatal/provincial, aun cuando aumentara el gasto al sector salud, sobre todo al hablar de la reestructuración del gasto público que se plantea para años futuros en cada sistema nacional y/o estatal de salud.

\section{REFERENCIAS}

1. Cercone J. Asociación público-privada para la atención a la salud: el desafío de la reforma en salud. In: Foro de Europa y América sobre las Reformas del Sector Salud. Santiago de Chile: Banco Mundial; 2002. p.6-11.

2. Gaziano TA. Chronic disease management in Latin America and Caribbean: Washington (DC): Inter-American Development Bank; 2011. p.54-68. Background paper prepared for RG-K1041 Prevention and Management of Chronic Disease. 
3. Wodon QT, Ayres R, Barenstein M, Hicks N, Lee K, Maloney W, et al. Poverty and policy in Latin America and the Caribbean. Washington (DC): The World Bank; 2000 [citado 30 sept 2017). (World Bank Technical Paper, 467). Disponible en: http://documents.worldbank.org/curated/ en/536291468774256064/Poverty-and-policy-in-Latin-America-and-the-Caribbean

4. Glassman A, Gaziano TA, Bouillon Buendia CP, Guanais de Aguiar FC. Confronting the chronic disease burden in Latin America and the Caribbean. Health Aff (Millwood). 2010;29(12):2142-8. https://doi.org/10.1377/hlthaff.2010.1038

5. World Health Organization. Guidelines to assess the social and economic impact consequences of the diseases. Geneva: WHO; 2009. p.37-56.

6. Arredondo A, Reyes G. Health disparities from economic burden of diabetes in middle-income countries: evidence from México. Plos ONE. 2013;8(7):e68443. https://doi.org/10.1371/journal.pone.0068443

7. Elliot WJ. The costs of treating hypertension: what are the long-term realities of cost containment and pharmacoeconomics? Posgrad Med. 1996;99(4):241-8, 251-2.

8. Snowdon A, Schnarr K, Hussein A, Alessi C. Measuring what matters: the cost vs. values of health care. London (CA): International Centre for Health Innovation; 2012 [citado 30 sept 2017]. p.12-20. Disponible en: http://sites.ivey.ca/healthinnovation/files/2012/11/White-Paper-Measuring-What-Matters.pdf

9. Johnson S, Delate T, Boka A, Shaw P, Zager C. Characterizing the financial burden of pulmonary arterial hypertension within an integrated healthcare delivery system. J Med Econ. 2013;16(12):1414-22. https://doi.org/10.3111/13696998.2013.849713

10. Secretaria de Salud (MEX). Encuesta Nacional Salud y Nutrición: Diabetes. 3.ed. México (DF): SSA; 2015. p.186-91.

11. Consejo Nacional de Población; Instituto Nacional de Estadística y Geografía. Censo de Población 2010-2015: indicadores sociodemograficos por estado. Mexico (DF): CONAPO; 2015.

12. Secretaría de Salud (MEX); Instituto Mexicano do Seguro Social; Instituto de Seguridad y Servicios Sociales de los Trabajadores del Estado. Casos de morbilidad hospitalaria por demanda específica, 1996-2013. Bol Inf Estad. 2014:57-81.

13. Arredondo A. Type 2 diabetes and health care costs in Latin America: exploring the need for greater preventive medicine. BMC Med. 2014;12:136. htttps://doi.org/10.1186/s12916-014-0136-z

14. Aitikin M. Statistical modelling and statistical inference: measurement error in the explanatory variables. Box-Jenkins technique. In: Aitikin M, Francis B, Hinde J. Statistical modelling in GLIM 4. 2.ed. New York: Oxford University Press; 2005. Chapter 2; Statistical modelling and statistical inference: measurement error in the explanatory variables. Box-Jenkins technique; p.112-32.

15. Banco de México. Indice Nacional de Precios por Servicios Médicos en México: Base 2000=100: la actividad económica en México: 1992-2014. México (DF): BM Gerencia de Investigación Económica; 2015. p.36-49. (Cuadernos Mensuales).

16. Candian Diabetes Association. An economic tsunami: the cost of diabetes in Canada. Toronto; 2009 [citado 30 sept 2017]. Disponible en: https://www.diabetes.ca/CDA/media/documents/publicationsand-newsletters/advocacy-reports/economic-tsunami-cost-of-diabetes-in-canada-english.pdf

17. American Diabetes Association. Economic costs of diabetes in the U.S. in 2012. Diabetes Care. 2013;36(4):1033-46. https://doi.org/0.2337/dc12-2625

18. International Diabetes Federation. Diabetes Atlas 2015: executive summary. 7. ed. Brussels: IDF; 2015.

19. Barceló A, Aedo C, Rajpathak S, Robles S. The cost of diabetes in Latin America and the Caribbean. Bull World Health Organ. 2003;81(1):19-27. https://doi.org/10.1590/S0042-96862003000100006

20. Zhang P, Zhang X, Brown J, Vistisen D, Sicree R, Shaw J, et al. Global healthcare expenditure on diabetes for 2010 and 2030. Diabetes Res Clin Pract. 2010;87(3):293- 301. https://doi.org/10.1016/j.diabres.2010.01.026

21. Santos JC, Moreira TMM. Risk factors and complications in patients with hypertension/diabetes in a regional health district of northeast Brazil. Rev EsC Enferm USP. 2012;46(5):1125-32. https://doi.org/10.1590/S0080-62342012000500013

22. Barreto SM, Miranda JJ, Figueroa JP, Schmidt MI, Munoz S, Kuri-Morales PP, et al. Epidemiology in Latin America and the Caribbean: current situation and challenges. Int J Epidemiol. 2012;41(2):557-71. https://doi.org/10.1093/ije/dys017 
23. International Diabetes Federation. Diabetes health economics: direct cost to the health care sector. Brussels; 2012. p.13-5.

24. Peckham R. Contagion: epidemiological models and financial crises. J Public Health (Oxf). 2014;36(1):13-7. https://doi.org/10.1093/pubmed/fdt083

25. Arredondo A, Orozco E, Aviles R. Evidence on equity, governance and financing after health care reform in Mexico: lessons for Latin American countries. Saude Soc. 2015;24 Supl1:158-75. https://doi.org/10.1590/S0104-12902015S01014

Financiamiento: Fondo Sectorial Salud del Consejo Nacional de Ciencia y Tecnología de México (Proyecto 0201306, año 2014).

Contribución de los Autores: Diseño del estudio, primer borrador del manuscrito, revisión de la literatura, análisis e interpretación de datos y revisión crítica: AA y EO. Colecta de datos, Análisis estadístico, análisis e interpretación de datos, revisión crítica del texto: AA, JAR, JN. Revisión de literatura, análisis e interpretación de datos: AAz, JN. Análisis de datos, revisión crítica del manuscrito: AA, EO, JAR, JN, AAz. Todos los autores aprobaron la versión final y se responsabilizan por el contenido del artículo.

Conflicto de Intereses: Los autores declaran no tener conflicto de intereses. 